\title{
Sensitivity Analysis of Detect and Avoid Well Clear Parameter Variations on UAS DAA Sensor Requirements
}

\author{
Jeremy Hardy ${ }^{1}$, Devin P. Jack ${ }^{2}$, and Keith D. Hoffler ${ }^{3}$ \\ Adaptive Aerospace Group, Hampton, Virginia, 23666
}

\begin{abstract}
In support of NASA's Unmanned Aircraft Systems Integration in the National Airspace System project and RTCA Special Committee 228, an analysis has been performed to provide insight in to the trade space between detect and avoid (DAA) Well Clear definition threshold variations, which could affect DAA sensor range and alerting requirements.
\end{abstract}

\section{Nomenclature}

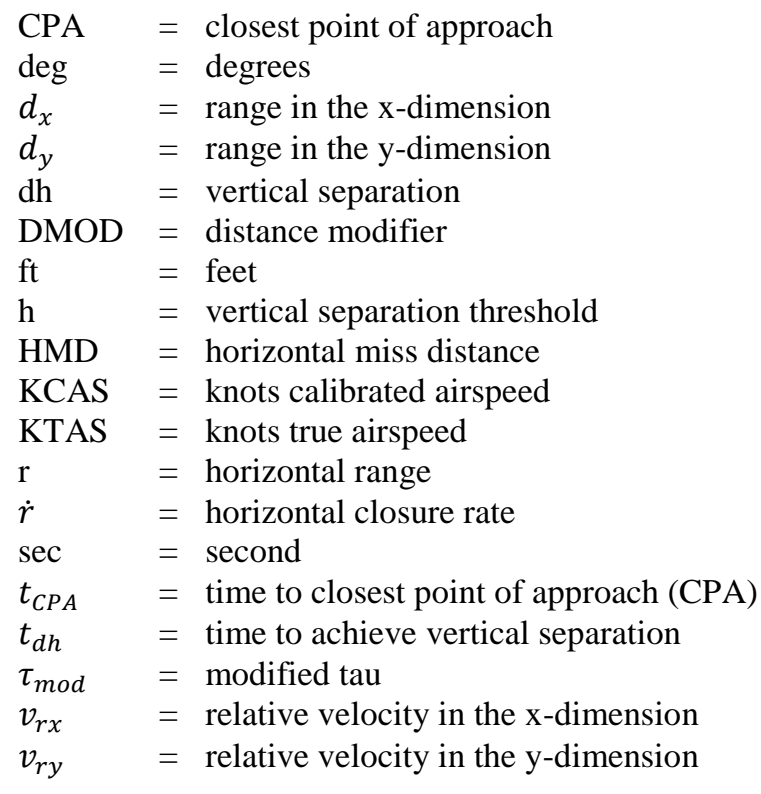

\section{Introduction}

The integration of unmanned aircraft systems (UAS) into the U.S.' National Airspace System (NAS) is highly desirable to many stakeholders across government, industry, and academia; maintaining the same level of safety as is currently in the NAS requires a means of replacing the manned aircraft pilot's obligation to see-and-avoid other aircraft [1]. RTCA Special Committee 228 (SC-228), a federal advisory committee consisting of public and private stakeholders, is developing minimum technical requirements for a detect-and-avoid (DAA) system to be used in lieu of a manned aircraft pilot's see-and-avoid capability. A DAA system uses a suite of sensors, trackers, detection algorithms, and display to provide the remotely located pilot with sufficient awareness to avoid a loss of separation with other aircraft. SC-228's recently published minimum operational performance standards (MOPS) for DAA systems are outlined in DO-365 [1], while the accompanying MOPS for air-to-air radar are outlined in DO-366 [2]. To make the problem more manageable, DO-365 and DO-366 were necessarily developed to support a limited set of UAS performance and operations. Ongoing work is intended to enable more types of UAS and support more complete operations.

\footnotetext{
${ }^{1}$ Research and Development Engineer, 100 Exploration Way Ste 330, Hampton, Virginia, 23666, Member.

${ }^{2}$ Research and Development Engineer, 100 Exploration Way Ste 330, Hampton, Virginia, 23666, Member.

${ }^{3}$ President and Senior R\&D Engineer, 100 Exploration Way Ste 330, Hampton, Virginia, 23666, Associate Fellow.
} 
The development of DO-365 made assumptions on the speed range and maneuverability of the unmanned aircraft (UA). In current NAS operations, a turn rate of 3 degrees per second is considered a standard-rate turn and is expected by controllers for most maneuvers. For most airspeeds in the NAS, this standard rate of turn keeps the accelerations experienced by the humans on-board to an acceptable level. To facilitate integration of UAS in the NAS, SC-228 asserted that UA must be capable of sustaining a turn at a rate no less than 3 degrees per second. The assumed speed range for DO_365 limited UA operations to between 40 and 200 KTAS while below 10,000 ft above mean sea level (MSL). Using these assumptions and an alerting timeline, range and time requirements were derived to define minimum sensor requirements.

The range requirement presented in DO-365 and DO-366 are driven by the 200 KTAS upper-bound, while the time requirement is driven by the lower bound of $40 \mathrm{KTAS}$. The upper bound was set in order to ensure that air-to-air radar could detect non-cooperative aircraft (aircraft without active surveillance systems) with sufficient distance to remain safely separated. The lower-end of the assumed speed range was defined to limit the need for excessively large alerting times; slow moving aircraft have difficulty achieving the needed separation. Currently, UAS must meet the requirements associated with these bounds. This work's companion paper [3] considers and presents the benefits to adjusting these performance requirements to benefit UAS with higher turning capabilities or providing reduced sensor or alerting requirements to accommodate UAS that can operate in more beneficial speed bands. In contrast, this paper explores the effects of changing DAA Well Clear (DWC) definition thresholds on minimum UA maneuver initiation range and time to LoWC, and describes an alternative method of analysis using contour plots to facilitate current RTCA SC-228 work to adjust the established DWC definition based on the operating airspace or UA performance[4].

\section{A. DAA Well Clear}

Per 14 CFR 91.113 [5], "vigilance shall be maintained by each person operating an aircraft so as to see and avoid other aircraft. When a rule of this section gives another aircraft the right-of-way, the pilot shall give way to that aircraft and may not pass over, under, or ahead of it unless well clear". Thus, utilizing an onboard pilot's ability to see and avoid traffic, an aircraft must remain 'well clear' from other vehicles at all times. With a pilot onboard, the definition of well clear is qualitative, based on the ability, judgment, and preferences of the pilot in command. Without an onboard pilot, a quantitative definition of well clear is required to establish separation requirements for unmanned aircraft DAA systems. A quantitative definition of well clear provides a repeatable target for which a DAA system may support safe separation from other aircraft by providing information to the pilot or to automation. The DAA Well Clear volume is intended to include interoperability principles with respect to Air Traffic Control (ATC) operations and current collision avoidance systems such as the Traffic alert and Collision Avoidance System (TCAS). To ensure interoperability with the current NAS, values used to define the well-clear volume must be large enough to avoid issuance of TCAS corrective resolution advisories but not so large as to interfere with ATC separation services [6]. This definition has been discussed and refined through a process involving NASA, Department of Defense (DoD), Federal Aviation Administration (FAA), and SC-228.

The definition of a DAA Well Clear volume is represented using the following inequality:

$$
\left[0 \leq \tau_{\text {mod }} \leq \tau_{\text {mod }}^{*} \text {. and. } H M D \leq H M D^{*}\right] \text {. and. }\left[-h^{*} \leq d h \leq h^{*}\right]
$$

where HMD is the kinematic projection of minimum horizontal miss distance in feet, and dh is the vertical separation between the two aircraft involved in the encounter. In Eqs. 1 through 4, the lack of a superscript denotes the instantaneous value at any given time during the encounter. The superscript $*$ denotes the value given as a minimum in the quantitative definition of well clear. Table 1 shows the numeric values used to define the DAA Well Clear threshold.

The definition of modified tau, $\tau_{m o d}$, is inherited from TCAS [7] and given in seconds by

$$
\tau_{\text {mod }}=\frac{D M O D^{2}-r^{2}}{\dot{r} r}
$$

where TCAS defines DMOD as a horizontal distance threshold with varying values depending on the ownship's altitude, range (r), and range rate $(\dot{r})$ between the two aircraft. For the purposes of the DAA Well Clear definition, the HMD* is used in place of DMOD. The predicted horizontal miss distance is represented as:

$$
H M D=\sqrt{\left(d_{x}+v_{r x} t_{C P A}\right)^{2}+\left(d_{y}+v_{r y} t_{C P A}\right)^{2}}
$$


where $d_{x}$ is the horizontal separation in the x-dimension, $d_{y}$ is the horizontal separation in the y-dimension, $v_{r x}$ is the relative horizontal velocity in the $\mathrm{x}$-dimension, and $v_{r y}$ is the relative horizontal velocity in the $\mathrm{y}$-dimension. The parameter $t_{C P A}$ is the time to closest point of approach (CPA) and is calculated as:

$$
t_{C P A}=\max \left(0,-\frac{d_{x} v_{r x}+d_{y} v_{r y}}{v_{r x}^{2}+v_{r y}^{2}}\right)
$$

Table 1 DAA Well Clear definition.

\begin{tabular}{|l|l|l|l|}
\hline Parameter & Symbol & Units & Value(s) \\
\hline Vertical Displacement & $\mathrm{h}^{*}$ & feet & 450 \\
\hline Modified Tau & $\tau_{\text {mod }}^{*}$ & seconds & 35 \\
\hline Horizontal Miss Distance & HMD* $^{*}$ & feet & 4000 \\
\hline
\end{tabular}

In the DWC definition in Eq. 1, the horizontal separation requirements are segregated from the vertical separation requirements. In other words, if an aircraft is adequately separated in the vertical plane to avoid a loss of DAA Well Clear (LoWC), the horizontal separation has no effect, and vice versa. Fig. 1 shows a simplified notional depiction of the DAA Well Clear definition, though its actual shape is more complex. The figure also separates vertical and horizontal separation requirements. In the horizontal dimension, both time and distance constraints must be violated in order to have a LoWC; whereas, in the vertical dimension, violation of only the distance constraint will result in a LoWC.

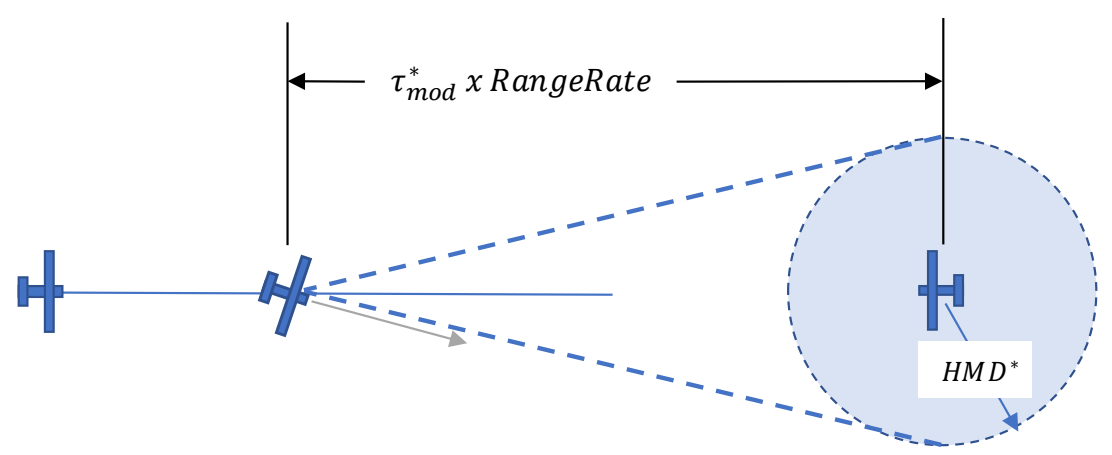

(a) Horizontal components of DAA Well Clear

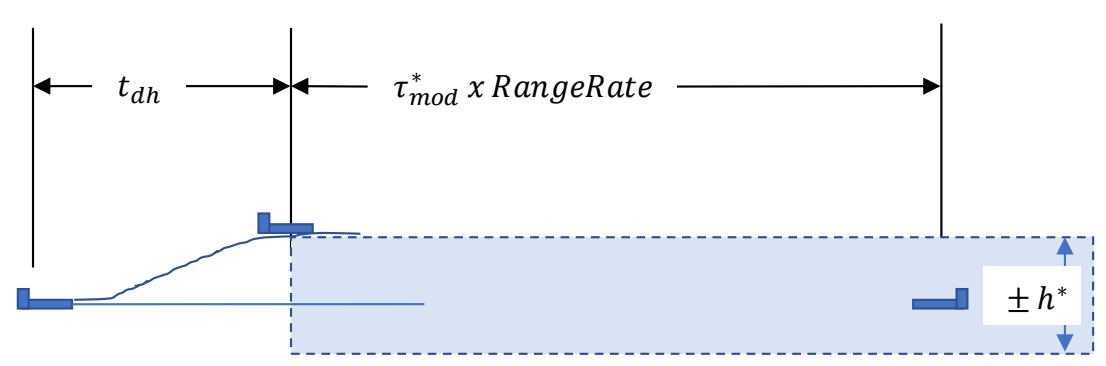

(b) Vertical components of DAA Well Clear

Fig. 1 Simplified depiction of DAA Well Clear

In the horizontal dimension, as shown in Fig. 1a the aircraft must remain outside of a time-based boundary ( $\tau_{m o d}^{*}$ ) unless the projected minimum distance between the two aircraft is greater than the specified HMD*. The HMD is a kinematic projection using the velocity vectors of the ownship and intruder aircraft. Thus, the maneuvering aircraft must turn to a heading that achieves a projected value of HMD greater than HMD* to achieve DAA Well Clear as 
opposed to being physically offset by a distance of HMD*. The angle that provides the required HMD projection varies greatly with the initial range between the encountering aircraft.

In the vertical plane, as shown in Fig. $1 b$, the aircraft must maintain spatial separation as defined by $\pm h^{*}$. In contrast to the horizontal separation requirements, the vertical separation is not a projection but an absolute vertical distance between the aircraft. Since the vertical boundary is fixed, the time required to reach the specified vertical separation $\mathrm{h}^{*}$, or $t_{d h}$, must be analyzed.

\section{B. Maneuver Initiation Reference Frame}

When defining range and time requirements for maneuvering to maintain DWC, it is important to consider the reference frame being used. The initial requirements presented in Appendix D of DO-365 [1] measure the range and time relative to the intruder aircraft as shown in Fig. 2a. This figure shows a notional depiction of the encounter geometry and DWC volume (dashed red). The figure indicates that the maneuver initiation range (MIR) and time to closest point of approach $\left(t_{C P A}\right)$ are measured relative to the intruder aircraft. Note that for simplicity the figure does not accurately depict the $t_{C P A}$ measurement, as this would more accurately be represented measured to the CPA point rather than the intruder position. This reference frame is of interest primarily for sensor measurements; an air-to-air radar directly measures the position of the intruder aircraft and has no means or interest in detecting a DWC volume.

In contrast to the intruder-centric measurements shown in Fig. 2a, the range and time may be measured relative to when the projected DAA Well Clear violation would occur. Fig. $2 \mathrm{~b}$ shows a notional depiction of the range and time measured relative to the DWC volume. This reference frame is preferable when examining time components of encounters because the DO-365 alerting requirements are generally provided as X seconds prior to loss of DAA Well Clear (LoWC). Thus, this reference frame is used for time components within the remainder of this paper and is

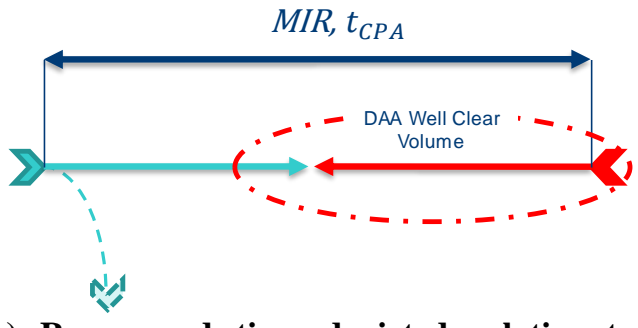

(a) Range and time depicted relative to intruder aircraft.

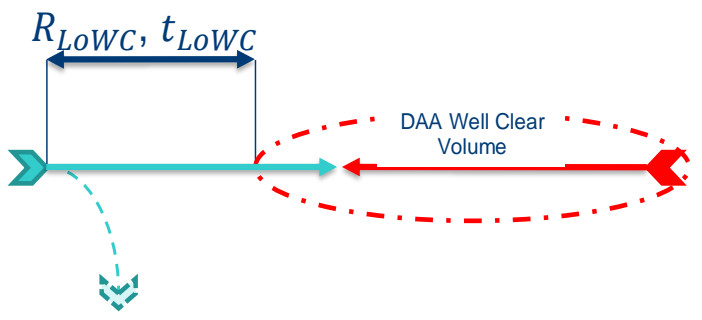

(b) Range and time depicted relative to DAA Well Clear Volume.

Figure 2 Reference frames for range and time measurements

proposed for subsequent versions of the DO-365 and related documents. Of these four parameter definitions, the two of primary interest are the range measured with respect to the intruder aircraft, MIR, and the time measured relative to the DWC volume, or time to loss of DAA Well Clear $\left(t_{\text {LoWC }}\right)$. The sensor range requirements can be derived from the MIR, and the alerting timeline contribution of the UAS maneuver may be assessed using the $t_{\text {LoWC }}$.

\section{General Approach}

\section{A. Encounter Description}

The 2-degrees-of-freedom Prototyping Aircraft Interaction Research Simulation (2PAIRS) [8] tool was used to analyze pairwise encounters involving a maneuvering UA and a constant trajectory non-cooperative intruder. The two aircraft are initially flying on a direct collision course $(\mathrm{CPA}=0$ feet). Initially trimmed to unaccelerated flight, the UA begins rolling in to the specified turn rate immediately at the start of the simulation. The UA maintains the specified rate of turn until leveling out when a heading change of 90 degrees is achieved. The scenario continues until CPA has occurred and the aircraft are diverging.

A limiting factor in the design of a DAA system is the range capability of the air-to-air radar, the primary means of detecting a non-cooperative aircraft (aircraft without active surveillance). The MIR is much less than the sensor capabilities for cooperative aircraft that actively broadcast their positions using ADS-B or an active surveillance transponder; therefore, cooperative intruders were not analyzed. For this reason, the intruder aircraft is modeled to be at the 95th percentile airspeed for non-cooperative aircraft in the NAS [9]. Further, the encounters are setup to occur at 10,000 feet MSL, the maximum operating altitude for non-cooperative aircraft [5]. Using the maximum operating altitude provides the highest true airspeed when converting from calibrated airspeed (such as the statute speed limit). 


\section{B. Experiment Design}

The findings of this paper were produced by generating aircraft encounters at discrete intervals varying the ownship's airspeed and the distance, through the variation of $t_{C P A}$, at which the aircraft in each encounter begin their head-on approach. Using the characteristics of each of these encounters DAA Well Clear variations were tested to find the required distance in order for the ownship to maneuver and maintain DWC in accordance with the definition being tested. This process yields the values MIR and $t_{\text {LoWC }}$ simultaneously.

Data presented in this paper are taken from encounters with an ownship constant turn rate of 3 degrees per second in accordance with minimum UAS requirements from DO-365 [1]. The UA airspeed was varied from 20-250 KCAS, or about $25-291$ KTAS at 10,000 ft MSL, which expands the operating airspeeds from the previous range in DO365. This range was chosen simply to test the extremes of practical and legal UA operation in the stated airspace and to support work currently being done to test the effects of both higher and lower speed UA as well as additional maneuverability [3]. The lower bound of $20 \mathrm{KCAS}$ is based on subject matter expertise and a survey of UA operator performance capabilities. The upper bound of $250 \mathrm{KCAS}$ is the statute speed limitation for aircraft below 10,000 feet MSL [5]. The UA airspeed was incremented by steps of 1 KCAS from 20 KCAS to 150 KCAS and then incremented in steps of 5 KCAS until reaching the upper limit of 250 KCAS.

The intruder aircraft was set to travel at a constant 170 KTAS which was found to be the $95^{\text {th }}$ percentile speed for non-cooperative aircraft [9]. Initial time to CPA, which effectively defines the initial range between the two aircraft as a function of the closure rate of the encounter, was independently varied between 1 and 125 seconds in 1 second intervals.

In addition to aircraft and encounter parameters, threshold values found in the logical definition of DAA Well Clear volume (see Eq. 1) were also varied; $\tau_{\text {mod }}^{*}$ values were varied between $0-35$ seconds in 5 -second increments, and HMD* was varied between 1500-4000 feet in 500-foot increments. Note the DO-365 definition of 35 seconds and 4000 feet is the upper bound of the parameter ranges of interest. This work focuses on the level-turn maneuver to maintain DWC, which is independent of the vertical DWC parameter, $\mathrm{h}^{*}$; therefore, no variations in $\mathrm{h}^{*}$ were considered.

\section{Results}

A selection of the results from the experiment are presented in the Figs. 3 and 4 where the MIR and $t_{\text {LoWC }}$ are plotted as a function of UA airspeed and selected DWC threshold values (HMD* and $\tau_{m o d}^{*}$ ). The selected subset of DWC threshold combinations were selected from the larger set of DWC definitions to present relevant information and a reasonable distribution for observation. The selected subset includes all combinations with $\tau_{m o d}^{*}$ of 0,15 , and 35

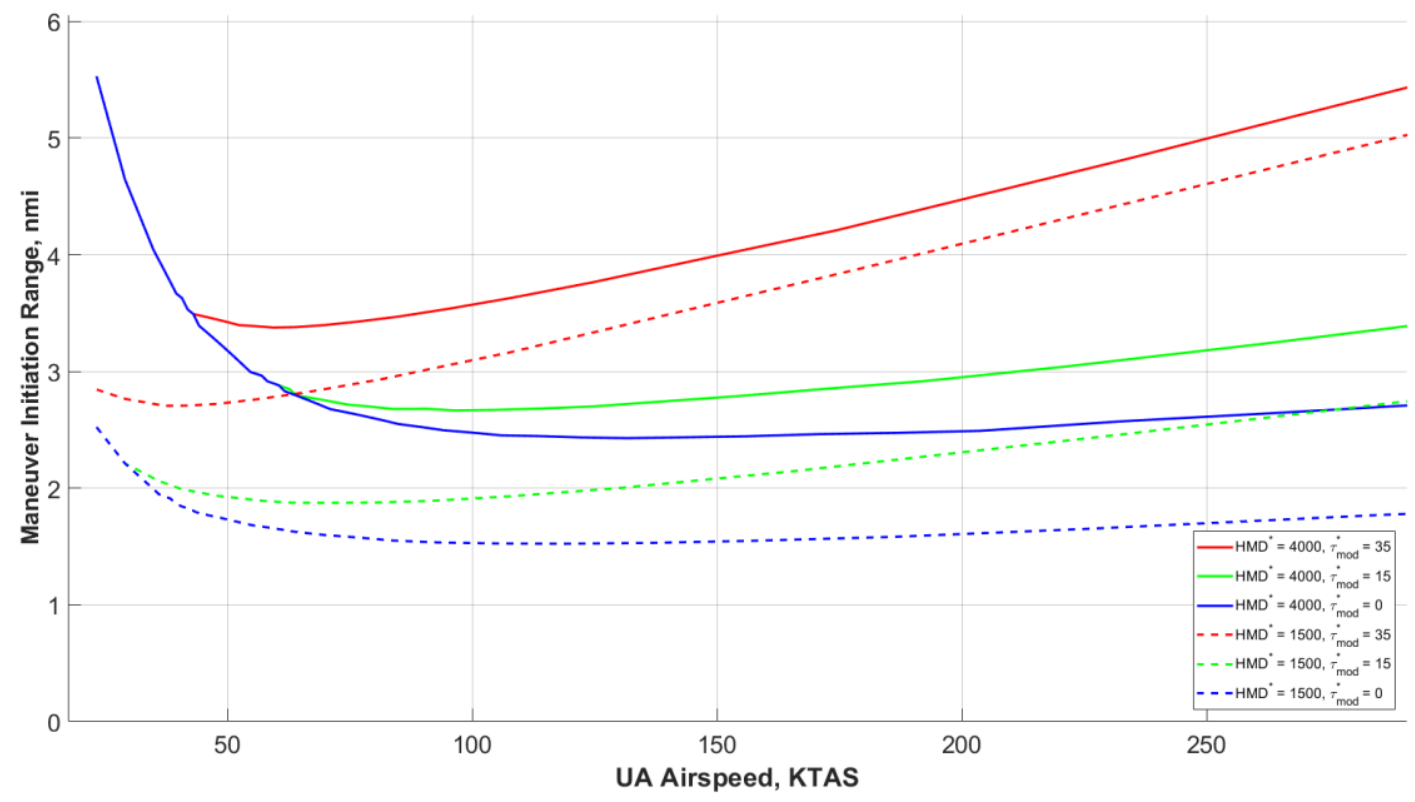

Fig. 3 Maneuver initiation range vs. UA airspeed of several DAA Well Clear definitions. 
seconds and HMD* values of 1500 and 4000 feet. The DO-365 values of 35 seconds and 4000 feet for $\tau_{m o d}^{*}$ and HMD*, respectively, are included. These values were selected as they are being considered for new DWC definitions for terminal airspace environment [10]. In the figures, color designates common $\tau_{m o d}^{*}$ values while line style (dashed vs. solid) designates common HMD* values. The solid red curve represents the DO-365 DWC definition [1].

\section{A. Maneuver Initiation Range}

From Fig. 3, the MIR values when defining HMD* as $4000 \mathrm{ft}$ and varying $\tau_{\text {mod }}^{*}$ (depicted as solid lines) overlap below approximately $40 \mathrm{KTAS}$, indicating that the MIR is independent of the $\tau_{\text {mod }}$ component of the DWC for $\tau_{\text {mod }}^{*}$ at and below the DO-365 value. Aircraft at low airspeed struggle to create the necessary lateral separation of HMD* in a reasonable amount of time to remain DWC. This results in no variation between all three 4000 feet HMD* definitions until the range-rate of the encounter becomes large enough that the 35 second $\tau_{\text {mod }}^{*}$ becomes the dominant factor in whether an encounter loses DWC. The divergence of the solid red line representing the DO-365 definition from the others indicates the airspeed at which the primary DWC parameter causing a LoWC shifts from HMD* below the indicated airspeed to $\tau_{m o d}^{*}$ for airspeeds greater than the indicated airspeed. For reference, the $\tau_{m o d}$ calculation is Eq. 2. The same trend is found for 1500 feet HMD* (dashed lines) with the exception that the velocity at which all three DWC definitions produce the same MIR occurs at an airspeed below the range of data analyzed for this experiment. This is because the HMD* component of the DWC is lower and thus, the $\tau_{m o d}^{*}$ component of DWC becomes the defining factor at lower speeds than when the HMD* value was higher.

When comparing DAA Well Clear definitions with similar $\tau_{\text {mod }}^{*}$, the shape of the curve, particularly the slope of the line at higher airspeeds, remains similar due to $\tau_{\text {mod }}$ dependency on range-rate as seen in Eq. 2 . The data also show that the magnitude of MIR is affected less by the decrease of HMD* when the $\tau_{\text {mod }}^{*}$ value of the definition is

Table 2 Minimum Achieved Maneuver Initiation Range and Corresponding KTAS.

\begin{tabular}{|c|c|c|c|}
\hline HMD* $(\mathrm{ft})$ & $\tau^{*}{ }_{\text {mod }}(\mathrm{sec})$ & Minimum MIR $(\mathrm{nmi})$ & KTAS MIR \\
\hline 4000 & 35 & 3.38 & 59 \\
\hline 4000 & 15 & 2.67 & 97 \\
\hline 4000 & 0 & 2.43 & 37 \\
\hline 1500 & 35 & 2.71 & 71 \\
\hline 1500 & 15 & 1.87 & 119 \\
\hline
\end{tabular}

higher. In other words, there is less variation in MIR between HMD* values of 4000 and 1500 feet when $\tau_{\text {mod }}^{*}$ is 35 seconds compared to the difference in MIR when $\tau_{\text {mod }}^{*}$ is 0 seconds.

The data provided in Table 2 contain the lowest maneuver initiation range for each of the six presented DAA Well Clear definitions and the UA airspeed at which the minimum MIR occurred. This data provide additional insight into Fig. 3 in terms of recognizing not only the precise changes in magnitude of MIR but also understanding the effect that reducing HMD* or $\tau_{\text {mod }}^{*}$ has on the shape of the resulting curve. As expected, reducing HMD* results in a reduction in the magnitude of the MIR. As mentioned previously, the magnitude of the change is dependent on the corresponding $\tau^{*}{ }_{\text {mod }}$ of the definitions being compared. The airspeed at which the minimum MIR is found is reduced as HMD* is lowered. The reduction of $\tau_{\text {mod }}^{*}$ also decreases the resulting MIR; however, this change to the DAA Well Clear definition moves the minimum MIR value to the right on the $\mathrm{x}$-axis so that as the value of $\tau_{\text {mod }}^{*}$ is lowered the optimal ownship airspeed gets larger. Table 2 also confirms what can be seen in the slope of the lines in Fig. 3 by comparing the change in the minimum MIR values and the UA airspeed at which the minimum occurs. Specifically, DAA Well Clear definitions with lower $\tau_{m o d}^{*}$ values have lower slope than those definitions with higher $\tau_{m o d}^{*}$ especially at the higher ends of the tested airspeed range. Reducing $\tau_{\text {mod }}^{*}$ for any DWC definition, would increase the range of speeds the UA could travel without drastically effecting the defining MIR. Characteristics of the standard DAA Well Clear definition, like the ones discussed above, are important to understand if the DAA Well Clear threshold values are to be adjusted to more effectively apply to different airspace operations, classes of aircraft with unique flight envelopes, or safety requirements.

\section{B. Time to Loss of Well Clear}

In addition to MIR, $t_{\text {LoWC }}$ required to maintain WC for each of the six DWC definitions is displayed in Fig. 4. Generally, the curves are similarly shaped across the tested DWC definitions: $t_{\text {LoWC }}$ monotonically decreases as the 
ownship airspeed increases. $\tau_{\text {mod }}^{*}$ effects the DWC volume by expanding the volume in the direction of the closure rate based on the magnitude of the closure rate. For a fixed closure rate and HMD*, lower $\tau_{m o d}^{*}$ results in smaller DWC volumes and thus larger values of $t_{\text {LoWC }}$. The inverse is true for HMD* where higher threshold values result in larger $t_{\text {LoWC }}$ values. The HMD parameter in the DWC definition is used as a means of filtering out encounters that

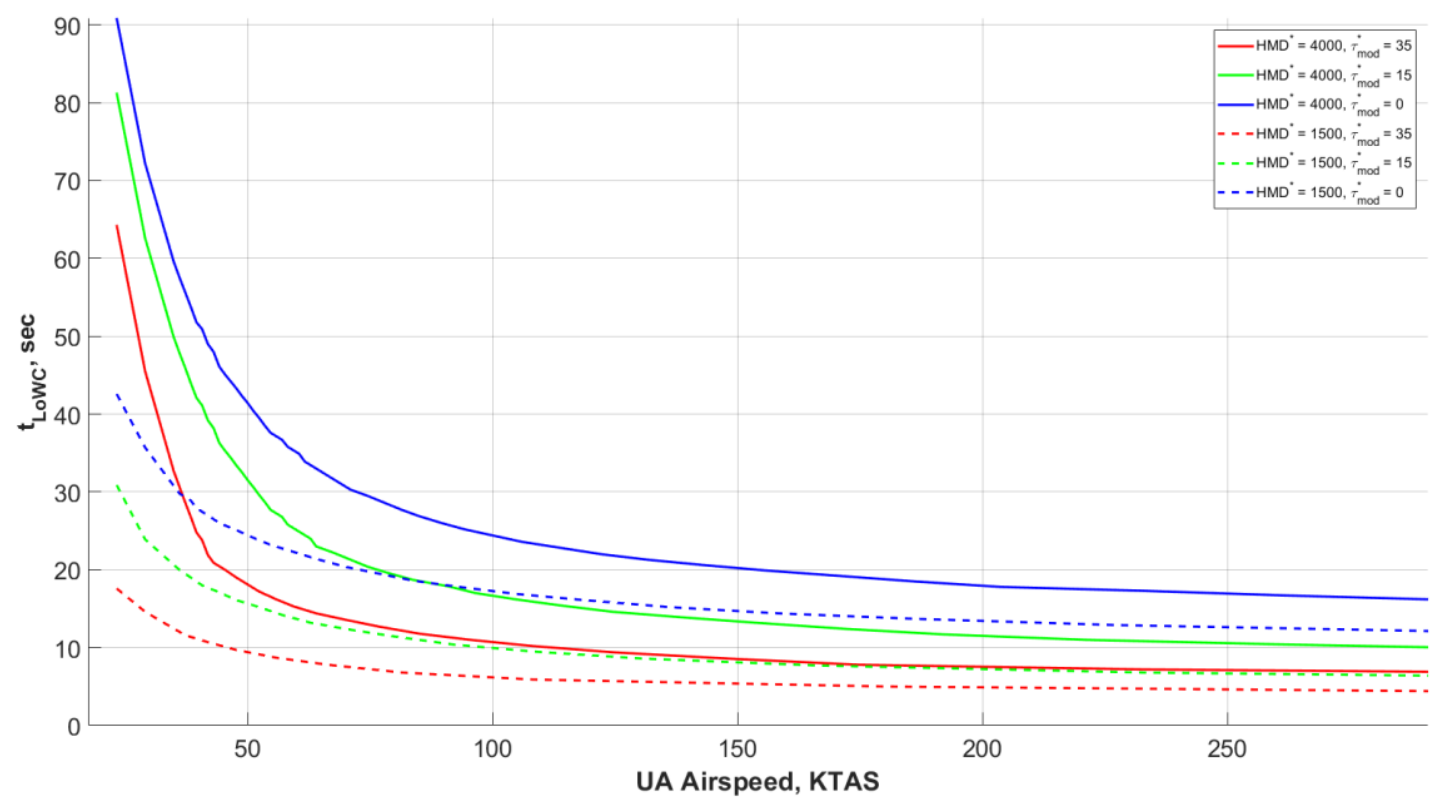

Fig. 4 Time to loss of DAA Well Clear vs. UA airspeed for several DAA Well Clear definitions.

are not projected to be within the specified minimum lateral separation of HMD*. For these head-on encounters, HMD* influences how far the UA must turn (and move for the slower UAs) to avoid a LoWC; the larger HMD* requires more time to turn thus the maneuver must begin earlier with respect to a LoWC. It is important when considering these trends to keep in mind that the encounters that define the MIR curve in Fig. 3 are the same encounters that define the $t_{\text {LoWC }}$ in Fig. 4, but the frame of reference for the data has changed, as discussed in Section 1B.

\section{Contour plot}

As an alternative method of analysis, contour plots were produced to analyze the effects of various DAA Well Clear definitions for aircraft that desire to carry low cost, size, weight, and power sensors [4]. A sample contour plot is shown in Fig. 5. The plot encompasses the defining (maximum) MIR values for encounters spanning from 20 KCAS to $250 \mathrm{KCAS}$ and for the corresponding DAA Well Clear threshold values on each axis. In the example shown, the defining MIR value always occurs at either $20 \mathrm{KCAS}$ or $250 \mathrm{KCAS}$, coinciding with the parabolic shape of MIR vs. UA airspeed in Fig. 3, where local maxima of MIR values occur at the extremes of the examined airspeed range. This tool is especially useful when the user is not concerned about the characteristics within a range of speeds, but only the limiting scenario of various DAA Well Clear definitions. 


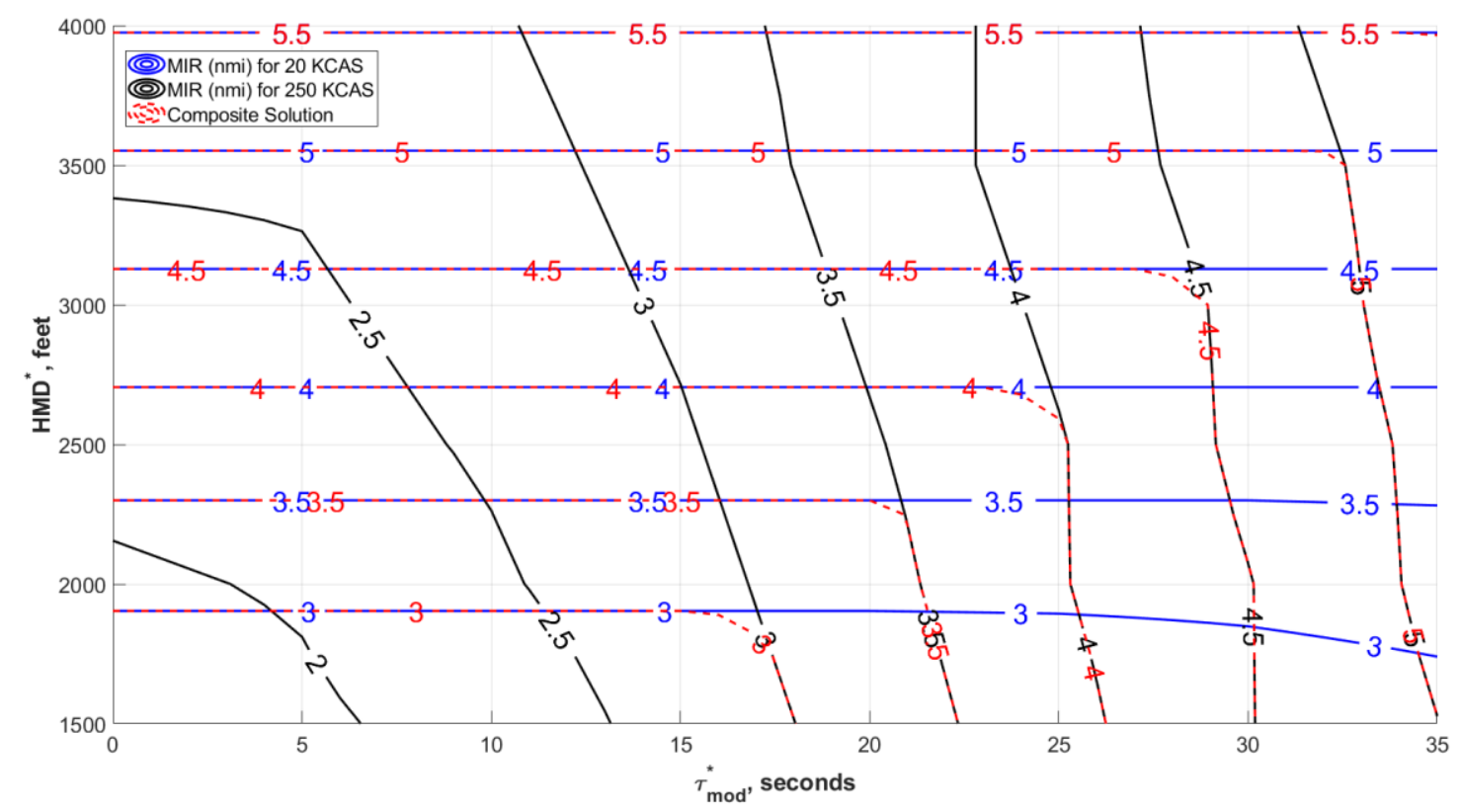

Fig. 5 Composite contour plot of MIR with respect to HMD and $\tau_{\text {mod }} 20$ KCAS to 250 KCAS.

\section{Conclusions}

Understanding which DWC parameters have significant effect on aircraft with various aircraft dynamics and sensor capabilities will contribute to finding safe alternatives to the current DWC definition that may allow for a wider variety of UAS to safely integrate in the NAS. While this experiment does not express which, if any, of the DWC parameter variations are safe for aircraft operations in the defined airspace, it displays trends and tradeoffs important to the decision-making process as well as an alternative analysis method that provides valuable information when considering changing the current definition. The effects of UA airspeed on MIR and $t_{\text {LoWC }}$ were discussed. For instance, it was shown that at low airspeeds the HMD* component of the DWC definition is the most influential on MIR and $t_{\text {LoWC }}$, but the associated low airspeed range where this behavior is seen is dependent on both HMD* and $\tau_{\text {mod }}^{*}$. Additionally, at these low airspeeds, the $t_{\text {LowC }}$ at which a UA must maneuver is shown to increase drastically versus high speed aircraft. The data and analysis presented herein is currently being used in the RTCA SC-228 community to determine DAA requirements associated with alternative DWC definitions for both low cost, size, weight and power sensors and terminal area operations with the intent of supporting a wide variety of UA and UAS operations in the NAS. 


\section{References}

[1] RTCA Special Committee 228, "DO-365: Minimum Operational Performance Standards (MOPS) for Detect and Avoid (DAA) Systems,” Standards DO-365, RTCA Inc., Washington, D.C., 2017.

[2] RTCA Special Committee 228, "DO-366: Minimum Operational Performance Standards (MOPS) for Air-to-Air RADAR for Traffic Surveillance,” Standards DO-366, RTCA Inc., Washington, D.C., 2017.

[3] Jack, D.P., Hardy, J., Hoffler, K.D., “Analysis of Influence of UAS Speed Range and Turn Performance on Detect and Avoid Sensor Requirements", 18th AIAA Aviation Technology, Integration, and Operations Conference, AIAA, June 2018.

[4] Wu, G. W., Cone, A. C., Lee., S., Edwards., M. W. M., and Jack, D. P., "Well Clear Trade Study for Unmanned Aircraft System Detect and Avoid with Non-Cooperative Aircraft," 18th AIAA Aviation Technology, Integration, and Operations Conference, AIAA, June 2018.

[5] Federal Aviation Administration, "14 CFR 91 General Operating and Flight Rules," Federal regulations, Department of Transportation, Washington, D.C., Apr. 2018. URL https://www.ecfr.gov/cgibin/retrieveECFR?gp=\&SID=de5f9357d1e2716832f6ca8b6f84244a\&mc=true\&n=pt14.2.91\&r=PART\&ty=HTML

[6] Consiglio, M., Chamberlain, J., Munoz, C., and Hoffler, K., 2013, 'Concept of Integration for UAS Operations in the NAS,' ICAS 2012-28th Congress of the International Council of the Aeronautical Sciences; 23-28 Sept. 2012; Brisbane, Australia.

[7] Federal Aviation Administration, "Introduction to TCAS II," version 7, November 2000.

[8] Jack, D. P., Hoffler, K. D., and Johnson, S.C., "Exploration of the Trade Space Between UAS Maneuver Performance and SAA System Performance Requirements," NASA CR-2014-218264, May 2014.

[9] Kochenderfer, M., et. al., "Uncorrelated encounter model of the National Airspace System version 1.0," MIT Lincoln Laboratory, Project Report ATC-345, 2008.

[10] Vincent, M., Trujillo, A., Jack, D., Hoffler, K., Tsakpinis, D., “A Recommended DAA Well-Clear Definition for the Terminal Environment", 18th AIAA Aviation Technology, Integration, and Operations Conference, AIAA, June 2018. 